\title{
Silver-lactoferrin nanocomplexes as a potent antimicrobial agent
}

\author{
Supporting Information
}

Paweł Pomastowski ${ }^{\ddagger}$, Mirosław Sprynskyy ${ }^{\ddagger}$, Petar Žuvela $^{\dagger}$, Katarzyna Rafińska $^{\ddagger}$, Maciej Milanowski ${ }^{\ddagger}$ J. Jay Liu ${ }^{\dagger}$, Myunggi $\mathrm{Yi}^{\infty}$, Bogusław Buszewski ${ }^{\ddagger}, *$

${ }^{\ddagger}$ Department of Environmental Chemistry and Bioanalytics, Faculty of Chemistry, Interdisciplinary Centre for Modern Technologies, Nicolaus Copernicus University, Toruń, Poland

${ }^{\dagger}$ Department of Chemical Engineering, Pukyong National University, 365 Sinseon-ro, Nam-gu, 608-739, Busan, Korea

${ }^{\infty}$ Department of Biomedical Engineering, Pukyong National University, 45 Yongso-ro, Nam-gu, 608-737, Busan, Korea

* Tel: +48 56 6114308; fax: +48 56 6114837.E-mail: bbusz@chem.umk.pl.

Electrophoretic analysis. Purified lactoferrin was analyzed using electrophoresis with a 4-12 \% gradient.

One single band of protein was observed between range of $98-62 \mathrm{kDa}$ (Figure S1).

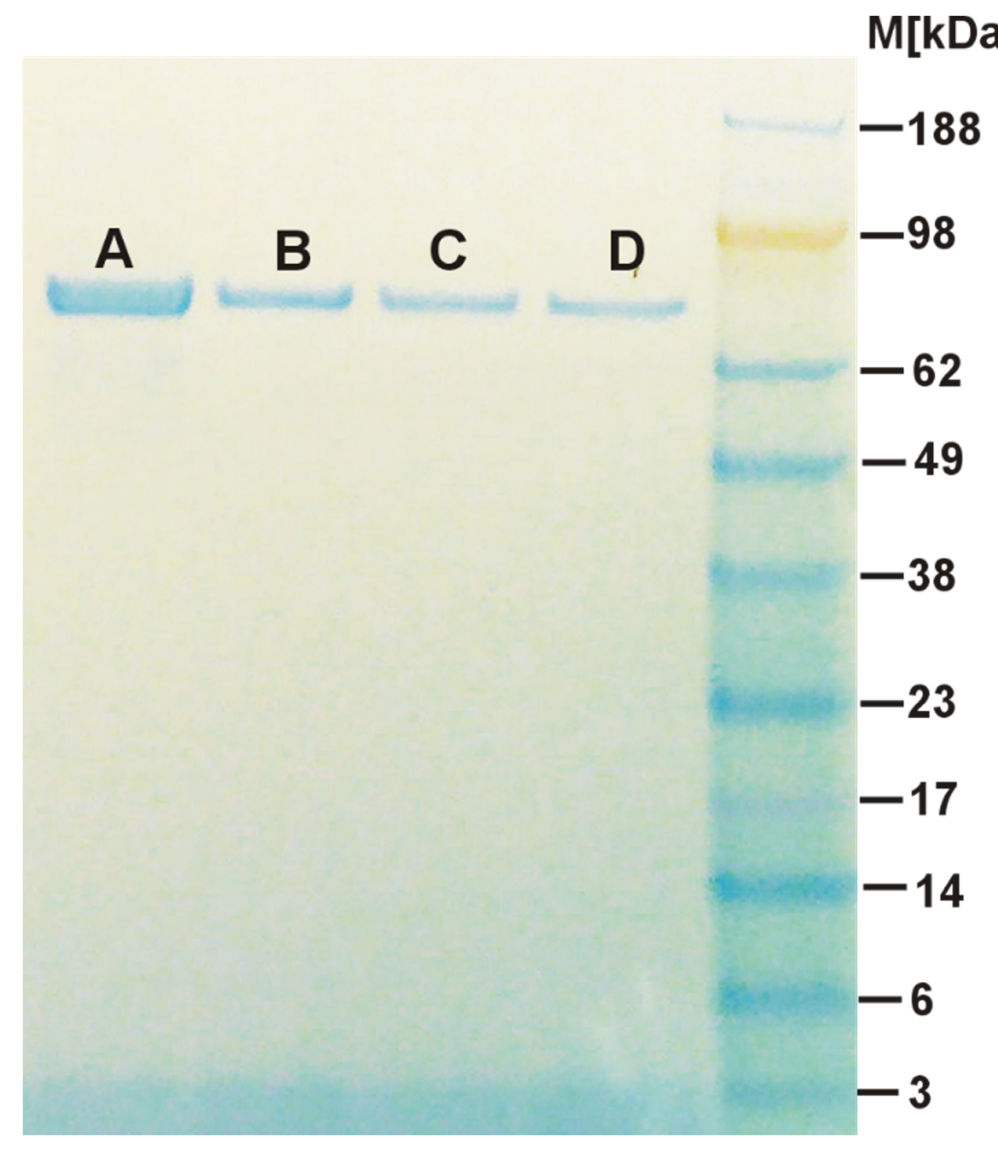

Figure S1. One-dimensional electropherogram of lactoferrin. Fraction of lactoferrin released under (A) $0.8 \mathrm{M} \mathrm{NaCl}$, and $0.7 \mathrm{M} \mathrm{NaCl}$ from (B) first, (C) second (D) and third (E) whey sample. $\mathrm{M}$ - protein marker. 
Spectroscopic analysis. Spectral characteristics of native and modified lactoferrin were evaluated in order to determine the active chemical groups involved in the silver-binding process. Fourier transform-infrared and Raman spectra are presented in supplementary figures S2A-S2C, and S3A-S3E, respectively.

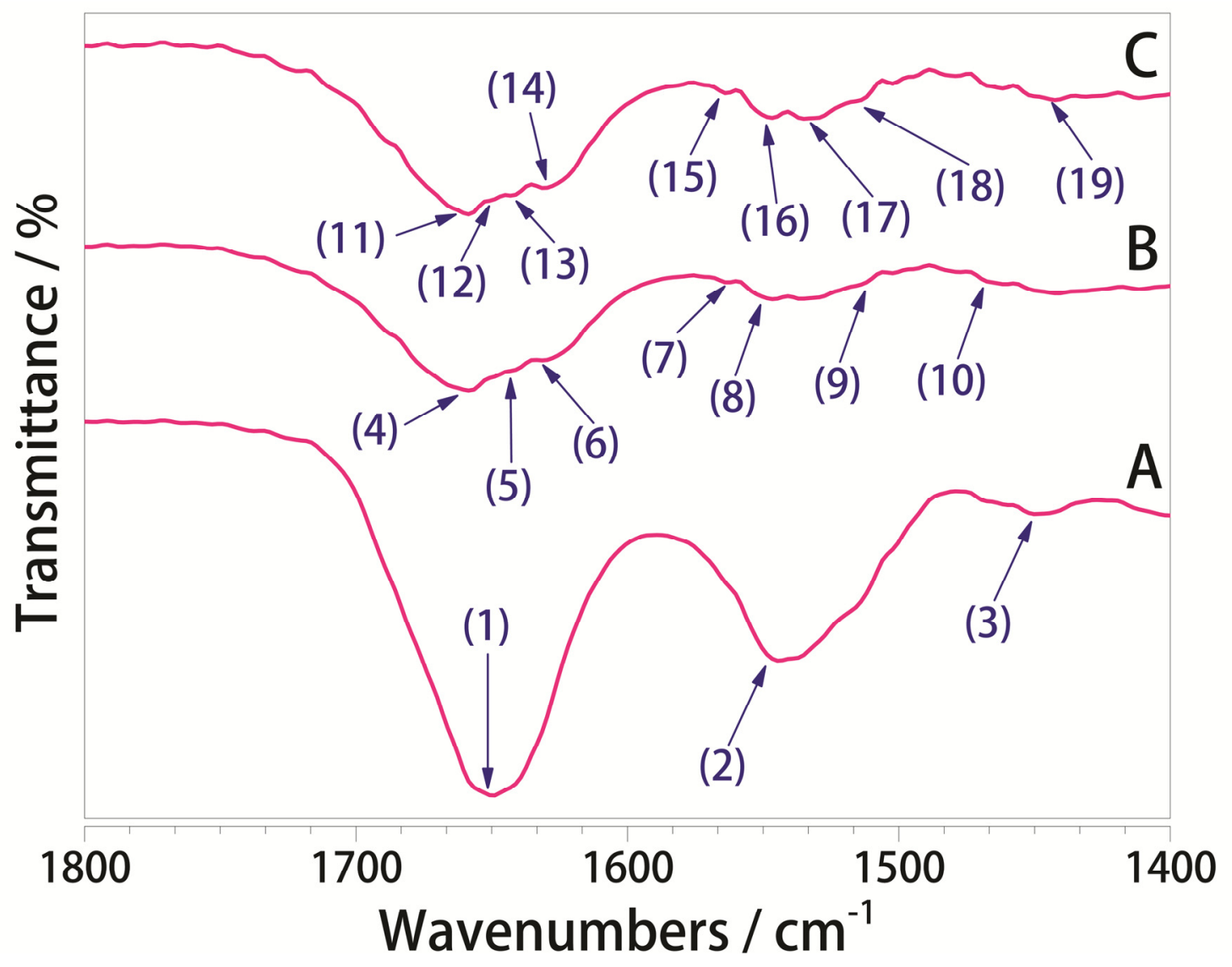

Figure S2. FT-IR spectra of native lactoferrin (A) before and after silver binding reaction in dependence at the time of uptake, i.e. (B) within initial $5 \mathrm{~min}$ and (C) after $20 \mathrm{~min}$. Legend: (1) $1649.17 \mathrm{~cm}^{-1},(2)$ $1544.46 \mathrm{~cm}^{-1}$, (3) $1444.63 \mathrm{~cm}^{-1}$, (4) $1658.39 \mathrm{~cm}^{-1}$, (5) $1639.95 \mathrm{~cm}^{-1}$, (6) $1628.55 \mathrm{~cm}^{-1}$, (7) $1562.36 \mathrm{~cm}^{-1}$, (8) $1548.26 \mathrm{~cm}^{-1}$, (9) $1502.14 \mathrm{~cm}^{-1}$, (10) $1465.25 \mathrm{~cm}^{-1}$, (11) $1659.40 \mathrm{~cm}^{-1}$, (12) $1649.45 \mathrm{~cm}^{-1}$, (13) $1641.50 \mathrm{~cm}^{-1}$, (14) $1628.23 \mathrm{~cm}^{-1}$, (15) $1564.58 \mathrm{~cm}^{-1}$, (16) $1547.34 \mathrm{~cm}^{-1}$, (17) $1534.74 \mathrm{~cm}^{-1}$, (18) 1515.51 $\mathrm{cm}^{-1},(19) 1440.40 \mathrm{~cm}^{-1}$. 

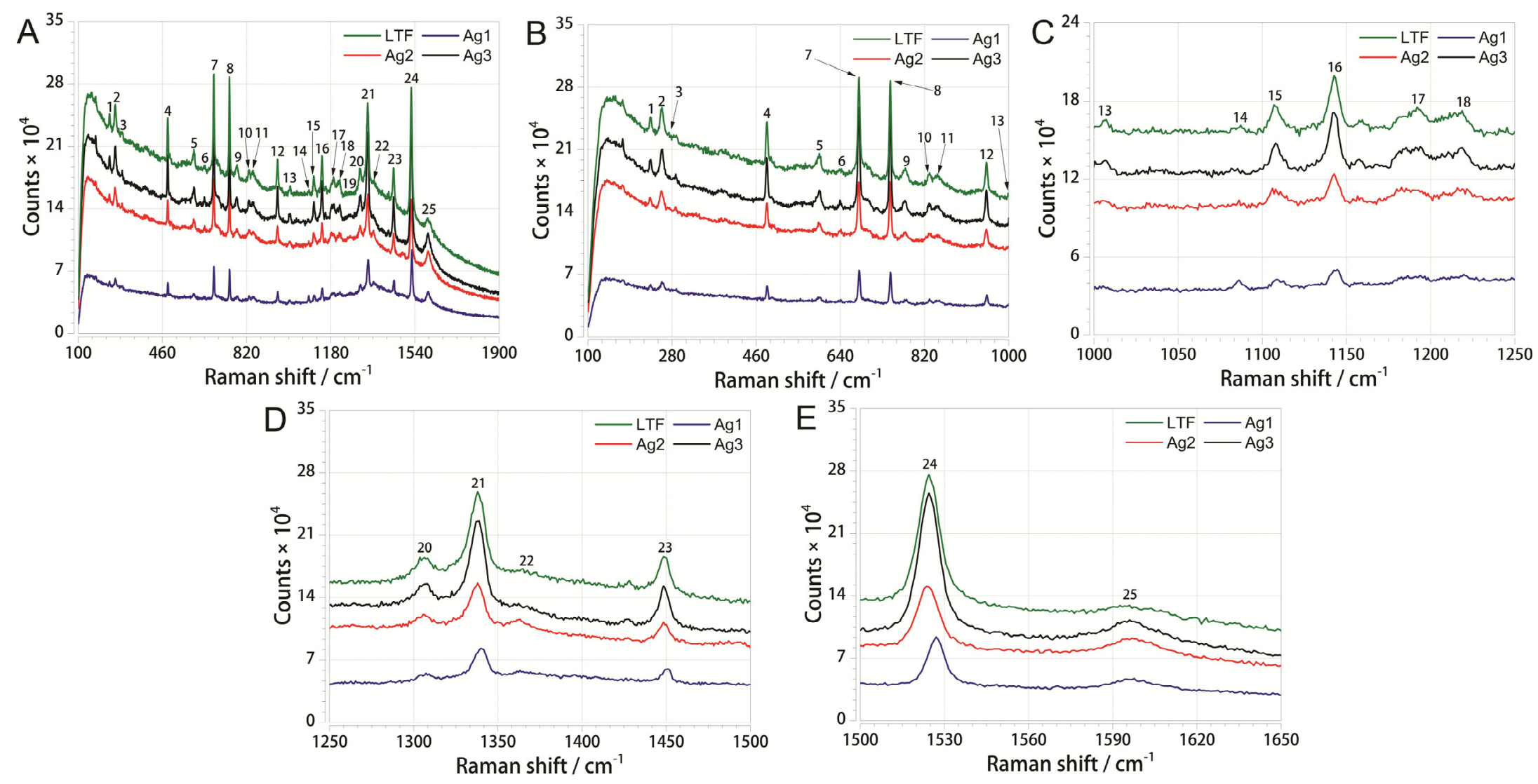

Figure S3. Raman spectra at the range (A) $100-1900 \mathrm{~cm}^{-1}$, (B) $100-1000 \mathrm{~cm}^{-1}$, (C) $1000-1250 \mathrm{~cm}^{-1}$, (D) $1250-1500 \mathrm{~cm}^{-1}$, and (E) $1500-1650 \mathrm{~cm}^{-1}$ for native lactoferrin (ltf) and after the process of binding silver cations dependent on the time of uptake, i.e. within 2 (Ag1), 3 (Ag2) and 20 (Ag3) minutes. Legend: (1) $238 \mathrm{~cm}^{-1}$, (2) $261 \mathrm{~cm}^{-1},(3) 292 \mathrm{~cm}^{-1},(4) 480 \mathrm{~cm}^{-1},(5) 593 \mathrm{~cm}^{-1},(6) 639 \mathrm{~cm}^{-1}$, (7) $681 \mathrm{~cm}^{-1}$, (8) $742 \mathrm{~cm}^{-1}$, (9) $780 \mathrm{~cm}^{-1},(10) 837 \mathrm{~cm}^{-1}$, (11) $846 \mathrm{~cm}^{-1}$, (12) $950 \mathrm{~cm}^{-1}$, (13) $1005 \mathrm{~cm}^{-1}$, (14) $1088 \mathrm{~cm}^{-1}$, (15) $1109 \mathrm{~cm}^{-1}$, (16) $1140 \mathrm{~cm}^{-1},(17) 1190 \mathrm{~cm}^{-1},(18) 1210 \mathrm{~cm}^{-1},(19) 1250 \mathrm{~cm}^{-1}$, (20) $1308 \mathrm{~cm}^{-1}$, (21) $1340 \mathrm{~cm}^{-1}$, (22) $1362 \mathrm{~cm}^{-1}$, (23) $1451 \mathrm{~cm}^{-1}$, (24) $1526 \mathrm{~cm}^{-1}$, (25) $1596 \mathrm{~cm}^{-1}$. 
Antibacterial activity of silver-lactoferrin complexes. To compare antibacterial activity of LTF and complex Ag-LTF flow cytometry measurements of optical density, zones of inhibition and direct counting of living and dead cells were utilized. The results are summarized in Table S1.

Table S1. Antimicrobial study of Ag-LTF against selected pathogens.

\begin{tabular}{|c|c|c|c|c|c|c|c|c|c|c|c|c|c|}
\hline \multirow[t]{2}{*}{ Test } & \multirow[t]{2}{*}{ C, ug } & \multicolumn{2}{|c|}{ OD } & \multicolumn{8}{|c|}{$\mathbf{C F}$} & \multicolumn{2}{|c|}{ DDM } \\
\hline & & $\operatorname{avg} \mathrm{A}_{600}$ & \pm SD & Dead & \pm SD & All & \pm SD & Live & $\pm \mathrm{SD}$ & \%live & \%dead & $\mathbf{S}[\mathbf{m m}]$ & \pm SD \\
\hline \multicolumn{14}{|c|}{ Enterococcus faecalis ATCC 14506} \\
\hline \multirow[t]{2}{*}{ blank } & 0 & 0.84 & 0.02 & 4500 & 527 & 93167 & 371 & 88667 & 344 & 95.17 & 4.83 & 0 & 0 \\
\hline & 12.5 & 0.18 & 0.01 & 4733 & 122 & 5833 & 343 & 1100 & 212 & 18.86 & 81.14 & 5 & 1 \\
\hline \multirow[t]{2}{*}{ Ag-LTF } & 25 & 0.10 & 0.01 & 3600 & 714 & 4400 & 419 & 800 & 146 & 18.18 & 81.82 & 15 & 2 \\
\hline & 50 & 0.06 & 0.01 & 4800 & 237 & 5167 & 430 & 367 & 513 & 7.10 & 92.90 & 28 & 2 \\
\hline \multirow[t]{2}{*}{ LTF } & 50 & 0.80 & 0.02 & 2567 & 417 & 82423 & 230 & 79856 & 443 & 96.89 & 3.11 & 0 & 0 \\
\hline & \multicolumn{13}{|c|}{ Escherichia coli ATCC 25922} \\
\hline \multirow[t]{2}{*}{ blank } & 0 & 0.81 & 0.01 & 2200 & 120 & 103052 & 621 & 100852 & 610 & 97.87 & 2.13 & 0 & 0 \\
\hline & 12.5 & 0.38 & 0.01 & 12600 & 78 & 101750 & 323 & 89150 & 425 & 87.62 & 12.38 & 0 & 0 \\
\hline \multirow[t]{2}{*}{ Ag-LTF } & 25 & 0.11 & 0.00 & 26676 & 82 & 33876 & 119 & 7200 & 317 & 21.25 & 78.75 & 4 & 1 \\
\hline & 50 & 0.06 & 0.00 & 29345 & 316 & 37845 & 111 & 8500 & 294 & 22.46 & 77.54 & 12 & 1 \\
\hline \multirow[t]{2}{*}{ LTF } & 50 & 0.78 & 0.08 & 5823 & 875 & 104033 & 988 & 98210 & 545 & 94.40 & 5.60 & 0 & 0 \\
\hline & \multicolumn{13}{|c|}{ Pseudomonas aeruginosa ATCC 27853} \\
\hline \multirow[t]{2}{*}{ blank } & 0 & 0.98 & 0.01 & 10150 & 446 & 421650 & 213 & 411500 & 1124 & 97.59 & 2.41 & 0 & 0 \\
\hline & 12.5 & 0.20 & 0.01 & 9750 & 76 & 57602 & 97 & 47852 & 750 & 83.07 & 16.93 & 0 & 0 \\
\hline \multirow[t]{2}{*}{ Ag-LTF } & 25 & 0.10 & 0.01 & 2965 & 114 & 5365 & 121 & 2400 & 223 & 44.73 & 55.27 & 3 & 1 \\
\hline & 50 & 0.01 & 0.00 & 3750 & 94 & 5400 & 144 & 1650 & 492 & 30.56 & 69.44 & 9 & 1 \\
\hline LTF & 50 & 0.85 & 0.08 & 2565 & 217 & $\begin{array}{r}102421 \\
\text { MR }\end{array}$ & 975 & 99856 & 4300 & 97.50 & 2.50 & 0 & 0 \\
\hline \multirow[t]{2}{*}{ blank } & 0 & 0.91 & 0.03 & 12150 & 112 & 510150 & 1750 & 498000 & 1622 & 97.62 & 2.38 & 0 & 0 \\
\hline & 12.5 & 0.33 & 0.05 & 100785 & 278 & 131285 & 599 & 30500 & 957 & 23.23 & 76.77 & 0 & 0 \\
\hline \multirow[t]{2}{*}{ Ag-LTF } & 25 & 0.12 & 0.01 & 17452 & 320 & 64852 & 899 & 47400 & 745 & 73.09 & 26.91 & 6 & 1 \\
\hline & 50 & 0.04 & 0.01 & 1785 & 211 & 2530 & 699 & 745 & 258 & 29.45 & 70.55 & 35 & 2 \\
\hline \multirow[t]{2}{*}{ LTF } & 50 & 0.90 & 0.02 & 2567 & 624 & 601127 & 790 & 598560 & 943 & 99.57 & 0.43 & 0 & 0 \\
\hline & \multicolumn{13}{|c|}{ Staphylococcus aureus ATCC 11632} \\
\hline \multirow[t]{2}{*}{ blank } & 0 & 0.90 & 0.05 & 2863 & 235 & 328334 & 336 & 325471 & 856 & 99.13 & 0.87 & 0 & 0 \\
\hline & 12.5 & 0.63 & 0.04 & 36400 & 333 & 111614 & 123 & 75214 & 748 & 67.39 & 32.61 & 0 & 0 \\
\hline \multirow[t]{2}{*}{ Ag-LTF } & 25 & 0.18 & 0.01 & 53257 & 746 & 63111 & 114 & 9854 & 492 & 15.61 & 84.39 & 8 & 1 \\
\hline & 50 & 0.07 & 0.03 & 7512 & 153 & 8486 & 222 & 974 & 269 & 11.48 & 88.52 & 25 & 2 \\
\hline LTF & 50 & 0.90 & 0.02 & 1986 & 456 & 389438 & 290 & 387452 & 743 & 99.49 & 0.51 & 0 & 0 \\
\hline
\end{tabular}

where: C,ug - amount of LTF or Ag-LTF add to medium, OD - optic density method, CF - flow cytometry method, DDM - disc diffusion method, avgA600 - average value of absorbance measured at $\lambda=600 \mathrm{~nm}$, dead 
Antibacterial activity of antibiotics. In order to evaluate antibacterial activity of antibiotics flow cytometry measurements of optical density and direct counting of living and dead cells were utilized. The results are summarized in Table S2.

Table S2. Antimicrobial study of antibiotics against selected pathogens.

\begin{tabular}{|c|c|c|c|c|c|c|c|c|c|c|c|}
\hline \multirow[t]{2}{*}{ Test } & \multirow[t]{2}{*}{ C, ug } & \multicolumn{2}{|c|}{ OD } & \multicolumn{8}{|c|}{$\mathbf{C F}$} \\
\hline & & $\operatorname{avgA600}$ & \pm SD & Dead & \pm SD & All & \pm SD & Live & \pm SD & \%live & \%dead \\
\hline \multicolumn{12}{|c|}{ Enterococcus faecalis ATCC 14506} \\
\hline blank & 0 & 0.74 & 0.02 & 4500 & 527 & 93167 & 371 & 88667 & 344 & 95.17 & 4.83 \\
\hline vancomycin & 4 & 0.08 & 0.02 & 4800 & 237 & 5167 & 430 & 367 & 513 & 7.10 & 92.90 \\
\hline cefotaxime & 4 & 0.27 & 0.01 & 13833 & 54 & 62300 & 56 & 48467 & 151 & 77.80 & 22.20 \\
\hline \multicolumn{12}{|c|}{ Escherichia coli ATCC 25922} \\
\hline blank & 0 & 0.81 & 0.01 & 2200 & 120 & 103052 & 621 & 100852 & 610 & 97.87 & 2.13 \\
\hline \multirow{2}{*}{$\begin{array}{l}\text { amoxicillin+clavulanic acid } \\
\text { ampicillin }\end{array}$} & 4 & 0.05 & 0.00 & 32145 & 3 & 39395 & 3 & 7250 & 1 & 18.40 & 81.60 \\
\hline & 0.1 & 0.58 & 0.03 & 15600 & 23 & 91500 & 65 & 87100 & 23 & 82.95 & 17.05 \\
\hline \multicolumn{12}{|c|}{ Pseudomonas aeruginosa ATCC 27853} \\
\hline blank & 0 & 0.98 & 0.01 & 10150 & 446 & 421650 & 213 & 411500 & 1124 & 97.59 & 2.41 \\
\hline cefotaxime & 4 & 0.07 & 0.01 & 3750 & 4 & 5400 & 4 & 1650 & 1 & 30.56 & 69.44 \\
\hline clindamycin & 0.1 & 0.39 & 0.03 & 68700 & 5 & 327600 & 1 & 258900 & 234 & 79.03 & 20.97 \\
\hline \multicolumn{12}{|c|}{ MRSA } \\
\hline blank & 0 & 0.91 & 0.03 & 12150 & 112 & 510150 & 1750 & 498000 & 1622 & 97.62 & 2.38 \\
\hline clindamycin & 4 & 0.08 & 0.01 & 29100 & 41 & 37350 & 132 & 8250 & 91 & 22.09 & 77.91 \\
\hline metronidazole & 0.1 & 0.45 & 0.04 & 8200 & 455 & 33700 & 232 & 25500 & 431 & 75.67 & 24.33 \\
\hline \multicolumn{12}{|c|}{ Staphylococcus aureus ATCC 11632} \\
\hline blank & 0 & 0.90 & 0.05 & 2863 & 235 & 328334 & 336 & 325471 & 856 & 99.13 & 0.87 \\
\hline ciprofloxacin & 4 & 0.15 & 0.01 & 44400 & 9 & 45739 & 17 & 1339 & 9 & 2.93 & 97.07 \\
\hline metronidazole & 0.5 & 0.51 & 0.03 & 8767 & 40 & 57700 & 95 & 48933 & 55 & 84.81 & 15.19 \\
\hline
\end{tabular}


Computing the cumulative number of sorbed silver atoms per one lactoferrin molecule. In the initial stage (first kinetics model segment), silver cations exhibited adsorption interactions. Beside that, $\mathrm{Ag}^{+}$was reduced to an elemental form $\left(\mathrm{Ag}^{0}\right)$. Maximum sorption capacity of the first segment was $6.58 \mathrm{mg}$ silver per $\mathrm{g}$ of lactoferrin. Taking into consideration that the molar mass of silver is $107.87 \mathrm{~g} \mathrm{~mol}^{-1}$, whereas the average molar mass of lactoferrin determined by MALDI TOF MS spectrometry is $80678 \mathrm{~g} \mathrm{~mol}^{-1}$, the number of sorbed silver ions per one lactoferrin molecule was computed as follows:

$$
\frac{n(\text { Ag })}{n(\text { lactoferrin })}=\frac{\frac{6.58 \times 10^{-3} \mathrm{~g}}{107.87 \mathrm{gol}^{-1}}}{\frac{1 \mathrm{~g}}{80678 \mathrm{~g} \mathrm{~mol}^{-1}}}=4.92 \approx 5
$$

Hence, five silver atoms are sorbed per one lactoferrin in the initial kinetics segment. After the second, slower segment attributed to diffusion of silver cations into the pores of lactoferrin, upon attaining equilibrium sorption capacity was $8.1 \mathrm{mg} \mathrm{g}^{-1}$. The number of sorbed silver atoms per one lactoferrin molecule was computed as follows:

$$
\frac{n(\mathrm{Ag})}{n(\text { lactoferrin })}=\frac{\frac{8.10 \times 10^{-3} \mathrm{~g}}{107.87 \mathrm{~g} \mathrm{~mol}^{-1}}}{\frac{1 \mathrm{~g}}{78360 \mathrm{~g} \mathrm{~mol}^{-1}}}=6.08 \approx 6
$$

Here, six silver atoms were sorbed per one lactoferrin molecule.

1400

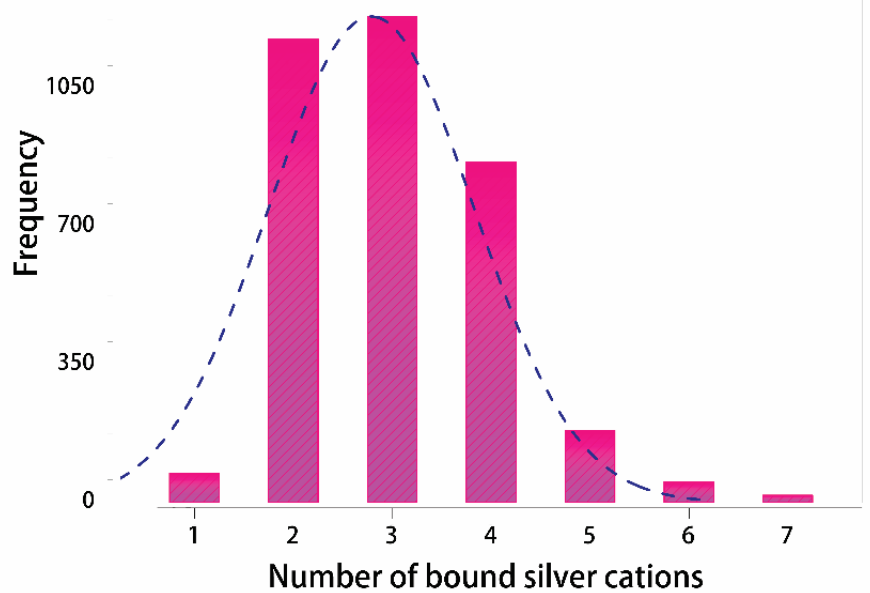

Figure S4. Histogram of frequency of silver cations' binding to lactoferrin. Sampling interval was $0.1 \mathrm{~ns}$, whereas the total simulation runtime was 36 nanoseconds. Sample mean of the number of bound silver cations was three. The threshold value of the distance between the centers of silver cation(s) and amino-acid residues involved in binding was $0.25 \mathrm{~nm}$. 

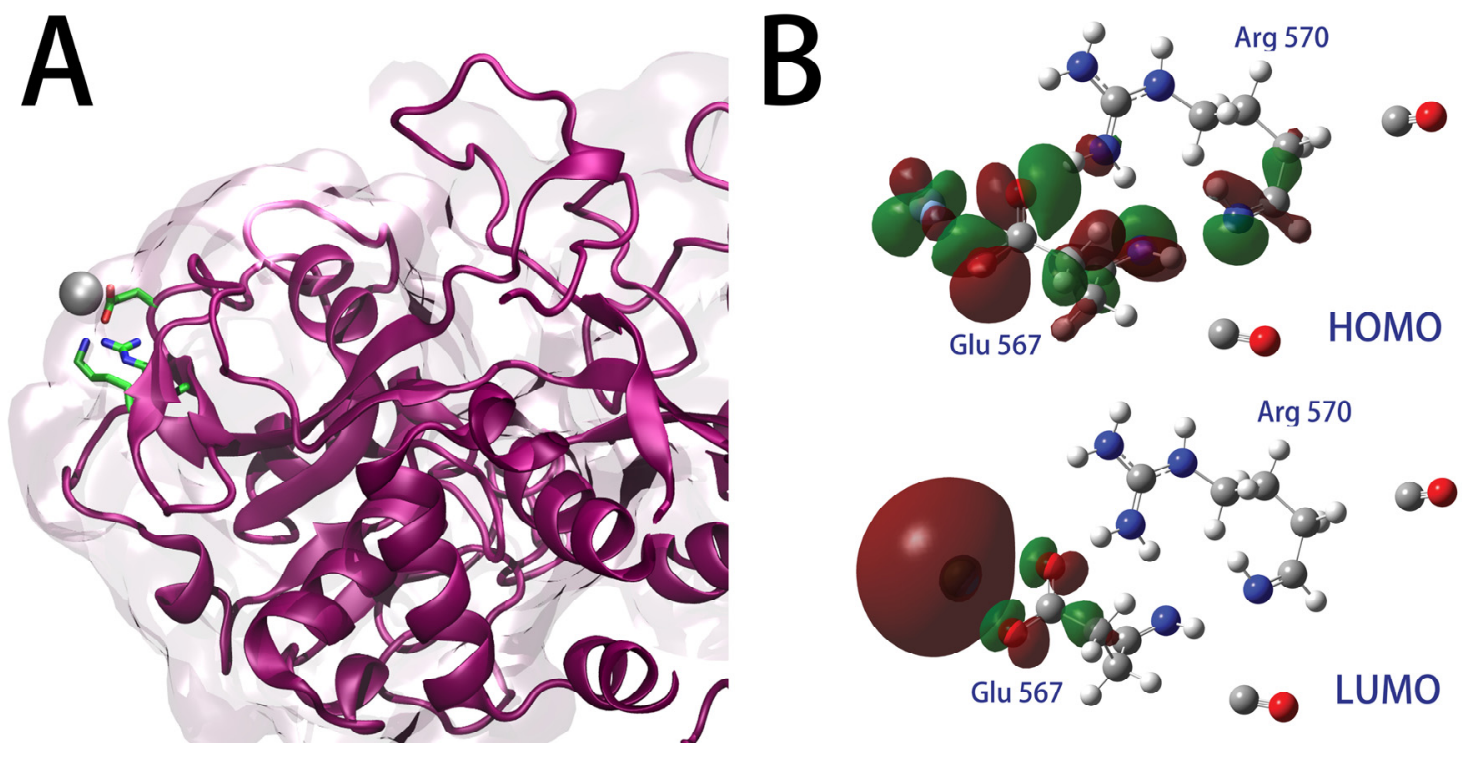

Figure S5. Molecular structure of lactoferrin with the silver cation bound to the (A) glutamic acid in the environment of arginine and lysine (MD snapshot), and (B) bound to Glu 567 in the environment of Arg 570 (QM snapshot). 


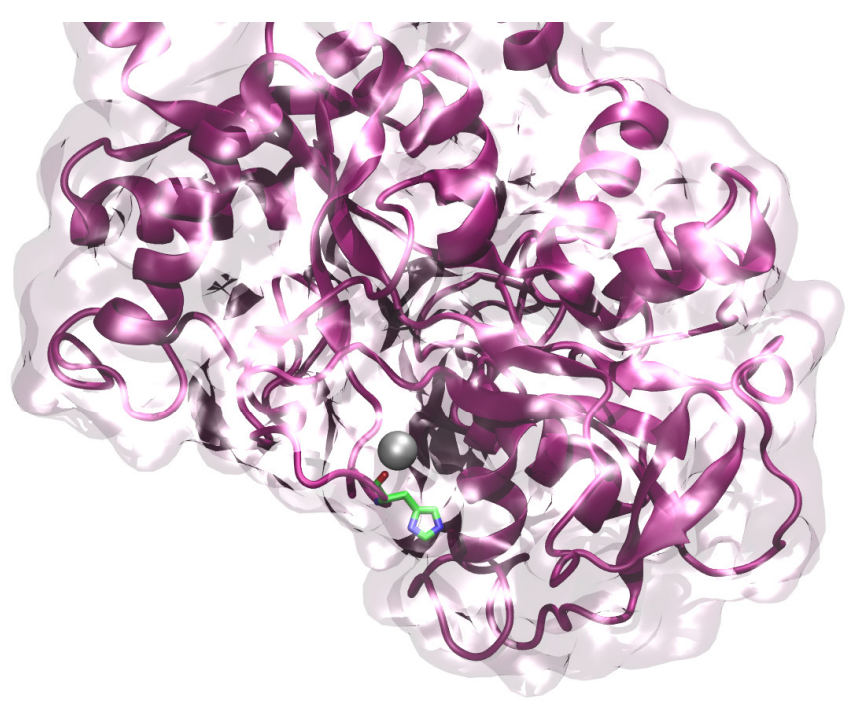

Figure S6. Molecular structure of lactoferrin with the silver cation bound to the carbonyl group of His 420 backbone. 